\title{
Differential response to shading in orthotropic and plagiotropic shoots of the clonal herb Glechoma hirsuta
}

Received: 23 December 1996 / Accepted: 19 July 1997

\begin{abstract}
Previous studies have shown that internodes and petioles of closely related erect and stoloniferous species show marked differences in their response to shading. Vertical structures show strong elongation responses while horizontal structures show significantly smaller elongation responses. This paper reports an experiment designed to test whether internodes and petioles on orthotropic (vertically oriented) and plagiotropic (horizontally oriented) shoots produced by the same plant, also show such differential responses. The study species, Glechoma hirsuta, produces plagiotropic shoots in its vegetative stage and orthotropic shoots during its generative stage. Shoots of G. hirsuta were grown either in full daylight or under simulated canopy shade. Internode and petiole elongation, biomass investment patterns and other growth-related parameters were measured on plants in each light treatment. In orthotropic shoots the length of internodes responded more strongly to shading than the length of petioles, while the opposite was true for plagiotropic shoots, confirming the hypothesis that vertical spacers have higher degrees of shade-induced plasticity than horizontally oriented spacers. Growth and development of horizontally oriented shoots was reduced by shading, whereas that of vertically oriented shoots was unaffected by light treatment. The results are discussed in terms of the differences in function of vertically and horizontally oriented spacers, and the probable benefits of plasticity in enhancing essential activities including photosynthesis, habitat exploration and seed dispersal.
\end{abstract}

H. Huber $(\bowtie)$

Department of Plant Ecology \& Evolutionary Biology,

Utrecht University, Sorbonnelaan 16,

3584 CA Utrecht, The Netherlands

e-mail: H.Huber@boev.biol.ruu.nl

M.J. Hutchings

School of Biological Sciences, University of Sussex,

Falmer, Brighton BN1 9QG, UK
Key words Biomass partitioning - Clonal growth · Glechoma Internode and petiole plasticity $\cdot$ Light availability

\section{Introduction}

The internodes and petioles of herbaceous plants project resource-acquiring structures (leaves and roots) into three-dimensional space during plant development. Internodes and petioles are frequently referred to as spacers (Bell 1984; Dong 1993, 1994). They may differ in the orientation of their growth depending on whether they develop on orthotropic or plagiotropic shoots, and, as a consequence, they may serve different ecological functions (Hutchings and de Kroon 1994; Huber 1996, 1997). For example, vertically oriented, orthotropic spacers (i.e. the internodes of erect shoots, and the petioles arising from stolons and rhizomes) raise leaf blades higher into herbaceous canopies, thereby increasing the likelihood of placing photosynthetic tissue in regions of higher light availability. Horizontally oriented internodes on plagiotropic shoots determine the positions of ramets (including their leaves and rooting points) in the horizontal plane (Birch and Hutchings 1992; de Kroon and Hutchings 1995). Petioles on orthotropic shoots may be inserted on the stems either horizontally or at various angles, and can fine-tune the positions of leaf laminae and their interception of photosynthetically active radiation by altering their angle of growth and/or their length (Monsi and Saeki 1953).

It has been shown for a number of closely related species that the extent of shade-induced plasticity in spacer length is related to the direction of growth of the shoot of which the spacer is a component, irrespective of the phylogenetic origin of the species. This suggests that different selection pressures may act on the spacers of plagiotropic and orthotropic shoots (Huber 1996; Huber et al., in press). Generally, vertically oriented spacers were found to exhibit greater plasticity in response to incident radiation than horizontally oriented spacers. 
These differences in plasticity were also associated with differences in biomass allocation patterns. A significantly higher proportion of biomass was allocated to spacers by vertically oriented shoots under shading than in full light. In contrast, allocation to spacers by horizontally oriented shoots was either not significantly changed or reduced by low light availability. It has been suggested that these differential responses of orthotropic and plagiotropic shoots to shading represent speciesspecific, potentially adaptive traits which may have evolved as a response to different patterns in the availability of light in the horizontal and vertical dimensions of natural habitats (Huber 1996; Stuefer 1996).

Some plant species can produce both plagiotropic and orthotropic shoots. The direction of growth of the main stem in such cases often depends on the developmental stage of the plant. For instance, in several species in the family Lamiaceae, vegetative shoots are plagiotropic and generative shoots are orthotropic. If the degree of plasticity in spacer length induced by shade is an invariant, species-specific trait, there should be no difference in the response to shading of internodes or petioles situated on differently oriented shoot types produced by the same plant. If, on the other hand, spacer plasticity is dependent upon shoot orientation, as in closely related species pairs (Huber et al., in press), differently oriented internodes or petioles on the two shoot types should show clear differences in their response to shading. This would imply that spacer plasticity is not an invariant trait within a plant, but dependent on the orientation of shoots and/or the developmental stage of the plant. This latter assumption forms the basis for the following hypotheses, which are tested in this study:

1. It is hypothesized that vertically oriented spacers will show proportionally more shade-induced plasticity in their length than horizontally oriented spacers, leading to the following predictions:

1a. Shade will induce proportionally more elongation in the internodes of orthotropic shoots than in those of plagiotropic shoots.

1b. Petioles of plagiotropic shoots will exhibit proportionally more plasticity in their length in response to shade than petioles of orthotropic shoots.

1c. In plagiotropic shoots, petioles will exhibit proportionally more plasticity in their length in response to shade than internodes on the same shoot type. Conversely, internodes of orthotropic shoots will show a proportionally stronger elongation response to shading than petioles on this shoot type.

2. Biomass investment in relative and/or absolute terms is expected to be positively correlated with spacer length. Under the assumption that hypothesis 1 is true, it is also hypothesized that biomass investment by shoots in vertical spacers should be greater, while investment by shoots in horizontal spacers should be lower in shaded than in high-light conditions.
To test these hypotheses, plagiotropic and orthotropic shoots of the herb Glechoma hirsuta (Lamiaceae) were grown in full daylight and under simulated canopy shade. The responses to light availability of spacer length, biomass allocation and growth related traits were compared in the two shoot types.

\section{Materials and methods}

Species

The stoloniferous herb Glechoma hirsuta Waldst. et Kit. (a close relative of $G$. hederacea L.) was used in this study. G. hirsuta is mainly found in deciduous forests in Eastern Europe (Hegi 1981). It produces two different shoot types. Plagiotropic shoots (i.e. long above-ground stolons) are formed in the vegetative stage (late spring to autumn). Generative shoots are orthotropic; flowering usually starts in March and can continue until May.

Orthotropic and plagiotropic shoots represent developmentally equivalent structures and are thus very similar in their modular construction. They are composed of (primary) modular units (sensu Watkinson and White 1986), each of which consists of a single node with an attached pair of leaves, and the internode preceding the node (Fig. 1). The meristems situated in the leaf axils of primary modular units can give rise either to lateral branches (in the case of plagiotropic shoots) or to flowers (in the case of orthotropic shoots; Fig. 1). In addition, lateral branches may originate from lower nodes on orthotropic shoots. Lateral branches consist of sequences of similar (secondary) modular units to those forming the main shoot.

The plant material used in the experiments was obtained from natural populations of $G$. hirsuta growing in a deciduous forest near Visegrad (Hungary) in April 1995. Plants were transferred to the botanical garden of Utrecht University, where they were propagated under quasi-optimal conditions in a common garden environment. The plants used in this study originated from seven different genotypes. Replicate plants multiplied from each genotype were allocated in equal numbers to each of the experimental treatments. However, due to the low number of replicates of each genetical individual within each treatment, genotype identity was not included as a factor in statistical analyses.

\section{The experiments}

The main aim of this study was to compare the degree of plasticity induced by shading in the lengths of spacers on orthotropic and

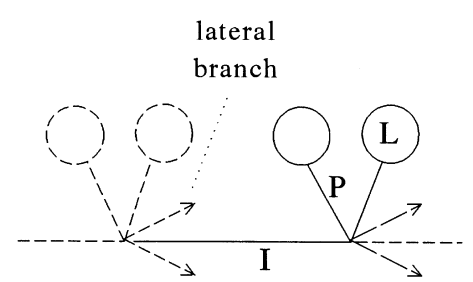

plagiotropic shoot

(vegetative stage)

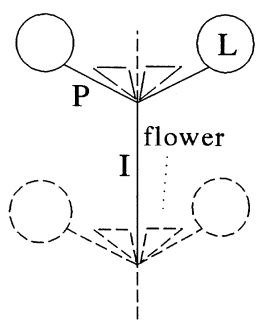

orthotropic shoot

(generative stage)
Fig. 1 Schematic illustration of the above-ground parts of orthotropic and plagiotropic shoots of Glechoma hirsuta. Solid lines are used to indicate all structural components contributing to a single modular unit. These consist of internodes $(I)$, petioles $(P)$ and leaf-laminae $(L)$ 
plagiotropic shoots. This was achieved by comparing the percentage change of spacer lengths produced by shoots of each type in high and low light treatments. In two separate experiments (subsequently referred to as E1 and E2), vegetative plants (i.e. those with plagiotropic shoots; E1) and generative plants (i.e. those with orthotropic shoots; E2) were grown under high and low light conditions. Since orthotropic and plagiotropic shoots are not produced at the same time in G. hirsuta, the two experiments could not be conducted simultaneously. Experiment E1 was performed in late summer 1995, while experiment E2 was carried out in spring 1996.

Both experiments were performed in a plastic greenhouse. Light availability inside the greenhouse was $85 \%$ of full daylight (measured with a Licor LI-185a light meter). This is subsequently referred to as $100 \%$ photosynthetic photon flux density (PPFD). In each of the experiments plants were subjected to two light treatments, (1) 100\% PPFD (ambient light conditions inside the greenhouse) with a red:far-red ratio of 1.09 , and (2) a simulated canopy shade treatment with $24 \%$ PPFD and a red:far-red ratio of 0.21 . Simulated canopy shade was used in this treatment, as morphological responses can be triggered by both changes in PPFD and in spectral light quality (red:far-red ratio). Shading was imposed by the use of shade cages covered by one layer of green plastic film (no. 122, fern green; Lee Colortran International, Hant, Hastings, UK), which reduced PPFD and red:far-red ratio to the values given above. In both experiments plants were provided with a non-limiting amount of nutrients, and watered daily with tap water.

\section{Experiment E1 (vegetative stage)}

In August 1995, apical cuttings bearing one or two rooted nodes and no secondary stolons were taken from plagiotropic shoots and transferred to the greenhouse. The cuttings were planted into trays $(100 \times 20 \times 20 \mathrm{~cm})$ filled with a 1:1 mixture of sand and sieved potting compost; $20 \mathrm{~g}$ of a controlled release fertilizer (Osmocote Plus, Sierra International, Heerlen, The Netherlands) was added uniformly to each tray. The nitrogen release amounted to $10 \mathrm{~kg} \mathrm{ha}^{-1}$ week $^{-1}$. Twelve cuttings were randomly assigned to each of the two treatments. The experiment lasted from 10 August to 20 September. Due to mortality the number of replicates at harvest was nine for the high-light treatment and ten for the shade treatment.

\section{Experiment E2 (generative stage)}

In spring 1996, orthotropic shoots were excised from stock plants and transferred to the greenhouse. These cuttings consisted of one rooted node and a small erect shoot bearing two or three internodes. Cuttings were planted into plastic pots $(12 \times 12 \times 12 \mathrm{~cm})$ filled with a 1:1 mixture of sand and sieved potting compost; $3 \mathrm{~g}$ of the controlled release fertilizer (see E1) was uniformly added to each pot. Fifteen replicates were subjected to each of the two light treatments. This experiment lasted from 27 March to 29 April 1996. Eleven plants per treatment survived until harvest.

\section{Measurements}

At harvest, parameters were measured which could be quantified for both plagiotropic and orthotropic shoots. Total length of primary shoots was measured and counts were made of the number of primary modular units (see Fig. 1), secondary branches and secondary modular units. Only secondary branches longer than $1 \mathrm{~cm}$ were counted (as in Birch and Hutchings 1992). The average weight of primary modular units was calculated by dividing the biomass of primary shoots (biomass of stems, petioles and leaf laminae combined) by the number of primary modular units.

For primary shoots the length and diameter of four consecutive internodes and petioles was measured. Internode and petiole measurements were made on nodes of developmentally similar ages. The four internodes were situated between the third and seventh nodes behind the apex. These internodes could be assumed to have finished elongation (Birch and Hutchings 1992). Length and diameter measurements were made on petioles on the third to sixth node behind the apex. For each node one of the two petioles was chosen randomly for measurement.

A sample of three leaves taken from five to seven nodes behind the apex of each replicate was used to measure area and weight of leaf laminae and petiole length and weight. These leaves could be considered to have finished expansion (Birch and Hutchings 1992). The remaining plant material was divided into primary internodes, petioles, leaf laminae and flowers, secondary structures, and roots. The dry weight of each of these plant parts was determined for each replicate after drying all plant material at $72^{\circ} \mathrm{C}$ for at least $48 \mathrm{~h}$.

\section{Statistical analyses}

The effects of shading on measured parameters were assessed separately for orthotropic and plagiotropic shoots, using one-way analysis of variance (ANOVA). Treatment effects on length and diameter of internodes were analysed by repeated-measures ANOVA with node position on the shoot as the repeated factor (Potvin et al. 1990; Dong 1993).

To test for differences in the degree of shade-induced plasticity between shoot types, orthotropic and plagiotropic shoots were compared by two-way ANOVA with shoot type and light-treatment as the main effects. Data obtained from measurements on four consecutive internodes and petioles were analyzed by two-way repeated-measures ANOVA with position on the shoot as repeated factor. In both of these analyses a significant interaction term indicates differences in plasticity between shoot types. In this study plasticity is defined as the relative difference (percentage change) in spacer length and in the values of other plant traits, between treatments. However, the general model underlying analyses of variance is based on additive effects (Sokal and Rohlf 1995). Therefore, log-transformations were performed prior to the analyses. This allows comparison by ANOVA of the relative, rather than absolute response to treatments (compare Huber 1996), because it transforms the multiplicative treatment effect (i.e. shadeinduced changes in spacer length and in the values of other parameters) into an additive effect.

As experiments E1 and E2 were not conducted simultaneously, growth-related parameters such as biomass and modular unit production of the two shoot types could not be directly compared. The statistical package SAS (SAS 1988) was used for all analyses.

\section{Results}

\section{Spacer length}

Internodes and petioles were significantly shorter on orthotropic shoots than on plagiotropic shoots in both light treatments (Fig. 2, Table 1B). The response of internode lengths to shading differed markedly between shoot types $(P<0.001$; Tables 1 and 2$)$. In plagiotropic shoots, mean internode length did not differ significantly between treatments, while internodes on orthotropic shoots were on average 55\% longer in shade than in high-light conditions (Fig. 2; Table 1).

In contrast, petiole plasticity did not differ significantly between shoot types (Tables 1 and 2). Petioles on plagiotropic shoots were approximately 50\% longer in shaded than in high-light conditions, while petioles on orthotropic shoots were $35 \%$ longer in shaded than in high-light conditions (Fig. 2, Table 2). The latter response was not statistically significant (Table 2). 
Table 1 Significance values obtained by repeated-measures analyses of variance on the length and diameter of internodes and petioles on four consecutive nodes with position as the repeated factor. Significance values are given for analyses performed for A plagiotropic and orthotropic shoots separately and for $\mathbf{B}$ the comparison between shoot types. The data are presented in Fig. 2.
Significance levels are as in Table 2. The most important comparisons are given in bold type. (Abbreviations used to indicate the statistical comparisons: $T$ treatment, $P$ position along the shoot, $S$ shoot type, plagiotropic or orthotropic. Variables are abbreviated as follows: $I L$ internode length, $P L$ petiole length, $I D$ internode diameter, $P D$ petiole diameter)
A Within shoot type

Plagiotropic
B Comparison between shoot types Orthotropic

\begin{tabular}{|c|c|c|c|c|c|c|c|c|c|c|c|c|c|}
\hline & $\mathbf{T}$ & $\mathrm{P}$ & $\mathrm{P} \times \mathrm{T}$ & $\mathbf{T}$ & $\mathrm{P}$ & $\mathrm{P} \times \mathrm{T}$ & $\mathrm{S}$ & $\mathrm{S}$ & $\mathbf{S} \times \mathbf{T}$ & $\mathrm{P}$ & $\mathrm{P} \times \mathrm{S}$ & $\mathrm{P} \times \mathrm{T}$ & $\mathrm{P} \times \mathrm{S} \times \mathrm{T}$ \\
\hline IL & ns & $* * *$ & $*$ & $* *$ & ns & $*$ & $* * *$ & $* *$ & $* *$ & $* * *$ & $* *$ & ns & $* * *$ \\
\hline PL & $* * *$ & $* * *$ & ns & ns & $* * *$ & ns & $* * *$ & $* *$ & ns & $* * *$ & $* *$ & ns & ns \\
\hline ID & $* * *$ & $* * *$ & ns & ns & $* * *$ & $*$ & ns & $* * *$ & $*$ & $* *$ & $* * *$ & $*$ & $*$ \\
\hline PD & ns & $* * *$ & ns & ns & $* * *$ & $*$ & $* * *$ & ns & ns & ns & $* * *$ & ns & ns \\
\hline
\end{tabular}
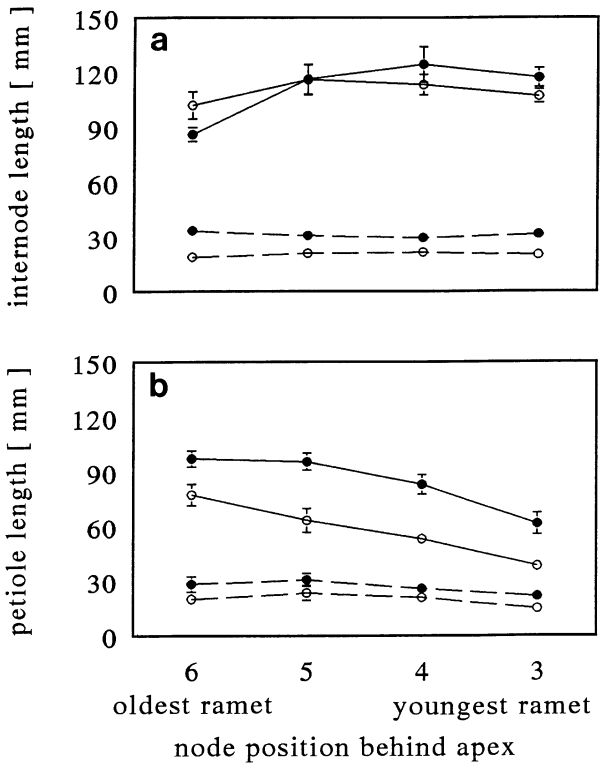

Fig. 2 Mean $( \pm 1 \mathrm{SE})$ lengths of four consecutive a internodes and b petioles on shoots of G. hirsuta. Solid lines indicate plagiotropic shoots and dashed lines orthotropic shoots. High-light treatments are represented by open circles and low-light conditions by filled circles. The numbers on the $x$-axis refer to the node position behind the apex. The node immediately behind the apex was given the number 1 . Standard errors of the means may be smaller than the symbols and therefore not visible. The results of the statistical analyses are given in Table 1

Table 2 Mean percentage increase in lengths of spacers produced in low-light and high-light conditions. Significance levels next to the values indicate whether the different types of spacing organs within plagiotropic or orthotropic shoots responded significantly to treatments. Significance levels next to the spacer name indicate whether the plasticity of each type of spacer differed significantly between shoot types. Significance indicators next to shoot type indicate whether petioles and internodes within one shoot type responded significantly differently to treatment

\begin{tabular}{lll}
\hline & Shoot type \\
\hline Spacer type & Plagiotropic*** & Orthotropic * \\
Internodes $* * *$ & $1.1 \% \mathrm{~ns}$ & $54.8 \% * *$ \\
Petioles & $48.5 \% *$ & $34.9 \% \mathrm{~ns}$ \\
\hline
\end{tabular}

*** $P<0.001, * * P<0.01,{ }^{*} P<0.05$, ns $P>0.05$

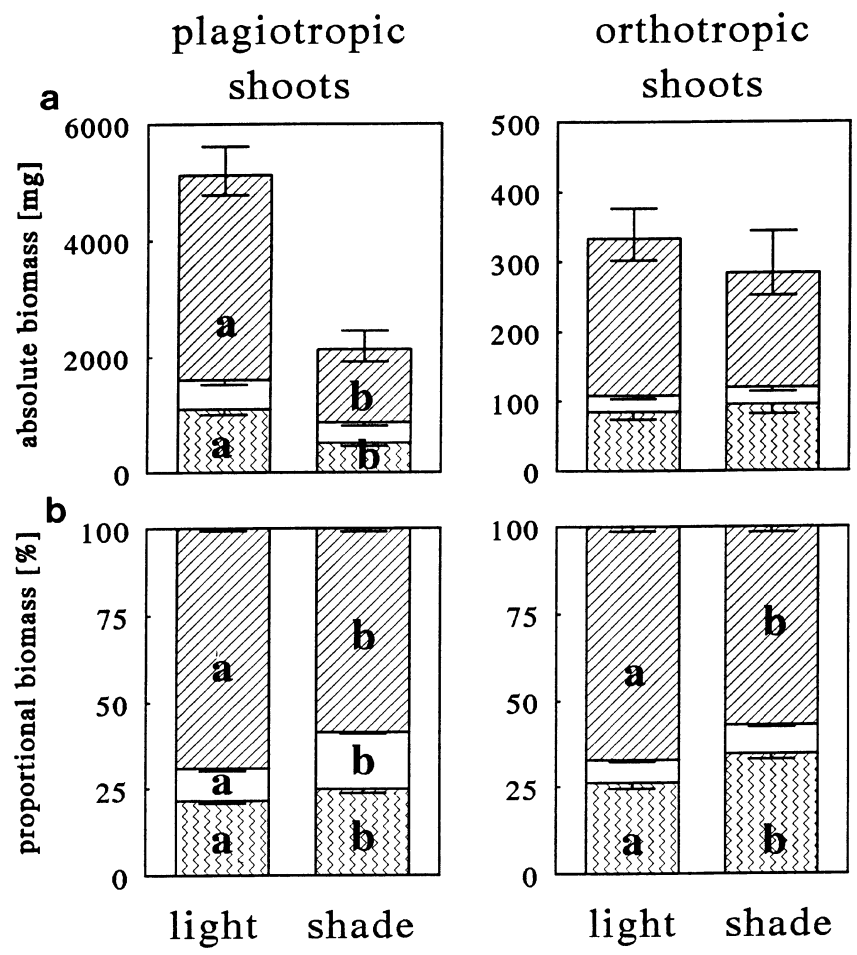

Fig. 3 a Absolute biomass of primary structures, and $\mathbf{b}$ proportional partitioning of the biomass of primary structures, to stems, petioles and leaf laminae (means $\pm 1 \mathrm{SE}$ ) in shoots of $G$. hirsuta. The graphs on the left give the values for the plagiotropic shoots and the graphs on the right values for orthotropic shoots. Different letters within bars indicate significant differences in the absolute and proportional allocation of biomass to different organs $(P<0.05)$. The results of the statistical comparisons between shoot types are given in Table 4

Internodes and petioles produced on plagiotropic shoots differed significantly in their response to shading (Table 2); elongation of petioles was significantly more pronounced under shade than was internode elongation $(P<0.001)$. The opposite pattern was found for orthotropic shoots, on which internodes showed a significantly stronger elongation than petioles in response to shading $(P<0.05$; Table 2). 
Table 3 Mean ( $\pm 1 \mathrm{SE}$ ) values for growth-related and morphological traits. Different letters next to the values indicate a significant response of orthotropic or plagiotropic shoots to light treatments at $P<0.05$. Significance values next to the variable name indicate whether shoot types responded differently to treatments. Significance values are as in Table 2

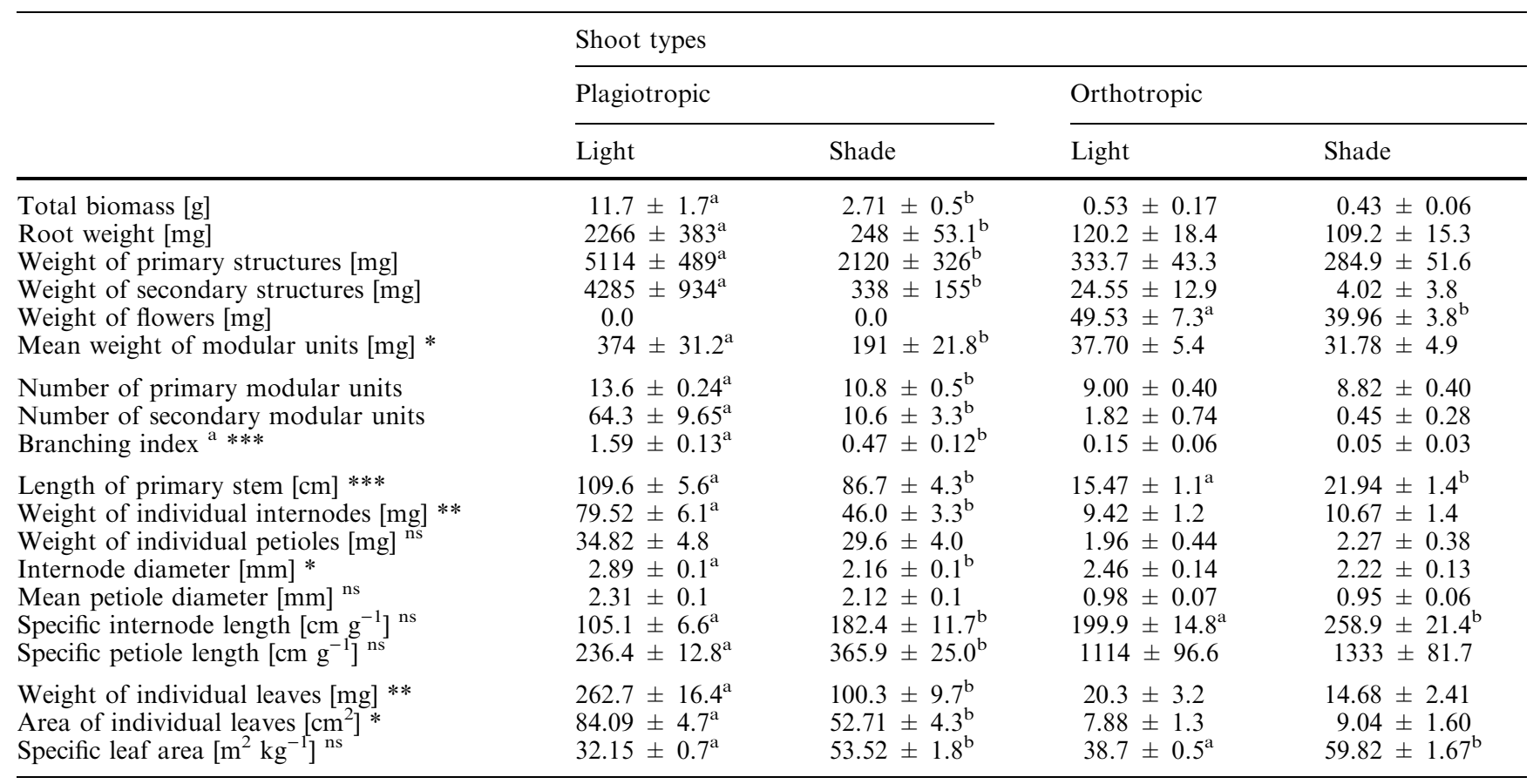

${ }^{a}$ The branching index was calculated as the number of branches divided by the nr of primary modular units

\section{Biomass investment in spacers}

The average weight of internodes showed a significantly different response to shading in the two shoot types (Fig. 3, Table 3). Shading significantly reduced the mean weight of internodes in plagiotropic but not in orthotropic shoots (Table 3 ). In contrast, the mean weight of petioles did not respond significantly to shading in either shoot type (Table 3).

Proportional allocation of shoot biomass (calculated for primary shoots only) to internodes did not differ significantly between shoot types. Allocation to internodes increased to a similar degree under shaded conditions in orthotropic and plagiotropic shoots (Fig. 3, Table 4). There was a significant difference between shoot types in response to light treatments in proportional allocation of biomass to petioles (Table 4). Shading led to a significant increase in proportional allocation of biomass to petioles in plagiotropic shoots, but not in orthotropic shoots.

\section{Additional spacer traits}

In plagiotropic shoots, shading resulted in the formation of significantly thinner internodes. In contrast, the diameter of internodes in orthotropic shoots did not differ significantly between treatments. Petiole diameter did not respond to shading in either shoot type (Tables 1 and 3 ).

The specific length (i.e. length per unit weight) of internodes and petioles showed similar responses to
Table 4 Significance values obtained by a two-way ANOVA with shoot type and treatment as the main factors. The table lists $P$-values which indicate whether the response of the respective organ to treatment differed between shoot types

\begin{tabular}{lll}
\hline \multicolumn{2}{l}{ Allocation of biomass within the primary stem } \\
\hline Spacer type & Absolute biomass & Relative biomass \\
\hline Internodes & 0.001 & 0.146 \\
Petioles & 0.276 & 0.007 \\
Leaf blades & 0.016 & 0.983 \\
\hline
\end{tabular}

shading in orthotropic and plagiotropic shoots (Table 3 ). In both shoot types specific internode length was significantly greater in shade than in high light conditions. In plagiotropic shoots specific petiole length was significantly greater in shaded than in high light conditions. This response was not significant for petioles on orthotropic shoots (Table 3).

\section{Growth-related parameters}

Total biomass and the biomass of roots and primary and secondary structures of plagiotropic shoots were all significantly reduced by shade (Fig. 3, Table 3). In orthotropic shoots, shading did not affect biomass production of any components except weight of flowers (Fig. 3, Table 3). The total length of primary shoots was significantly decreased by shading in plagiotropic shoots but significantly increased in orthotropic shoots (Table 3). 
Plagiotropic shoots produced significantly fewer primary modular units in shade than in high light (Table 3). In addition, the production of secondary branches was strongly reduced in shade. This led to an $80 \%$ decrease in the number of secondary modular units under these conditions $(P<0.001$, Table 3$)$. In orthotropic shoots the number of primary modular units, secondary branches and secondary modular units were not significantly affected by shading (Table 3 ).

\section{Discussion}

\section{Spacer plasticity}

This study provides evidence that plasticity of internode length in G. hirsuta depends on shoot orientation. Internodes on orthotropic shoots were $55 \%$ longer in shade than in high light, while those on plagiotropic shoots hardly responded to shading. This confirms our hypothesis that shade should induce a higher degree of elongation in the internodes of orthotropic shoots than in the internodes of plagiotropic shoots produced by the same species. This result matches earlier findings that in closely-related species pairs having shoots with different growth directions, stoloniferous species generally show lower degrees of shade-induced internode elongation than related species with erect shoots (Huber 1996; Huber et al., in press). The effects of shade on the length of orthotropic internodes of G. hirsuta were similar to the effects on erect species, and the effects of shade on internode length of plagiotropic shoots were similar to the effects on stoloniferous species. In G. hirsuta, orthotropic and plagiotropic shoots are produced at different stages in the life cycle. Our data thus provide evidence of a change in internode plasticity during the ontogeny of individual plants.

Shading led to a significant increase in petiole length in both shoot types, but the scale of the response was not significantly different. This contradicts our hypothesis that petioles of plagiotropic shoots should respond more to shade than petioles on orthotropic shoots. A comparative study of spacer plasticity in a large number of closely-related species with erect or stoloniferous shoots also failed to detect consistent differences in petiole plasticity (Huber et al., in press). A functional interpretation of this response pattern may be that light capture by leaf laminae can be enhanced not only by the elongation of internodes on orthotropic shoots but also by elongating petioles, provided that these do not emerge horizontally from the shoots. This may also explain the relatively high degree of shade-induced plasticity in petiole length in G. hirsuta.

Within shoot type, vertical spacers exhibited higher degrees of plasticity in their length than horizontal spacers, i.e. internodes on orthotropic shoots and petioles on plagiotropic shoots showed the most pronounced length responses to shading. This confirms the third part of our first hypothesis and matches results obtained for several other erect and stoloniferous species (Evans 1992; Dong 1993, 1995; Thompson 1993; Huber 1996, 1997).

These differences in plasticity in the length of spacers with different orientation may have evolved in response to environmental effects on the functioning of plant structures (Scheiner 1993; Huber 1996). The ability of vertically oriented spacers to raise leaves higher in the canopy by exhibiting plasticity, will usually enhance light interception (Ballaré 1994) because light intensity increases predictably in the vertical plane (Monsi and Saeki 1953; Tappeiner and Cernusca 1996). Relative light levels are less predictable in the horizontal plane, and therefore increases in the length of horizontal spacers will not always yield benefits (de Kroon and Hutchings 1995; Oborny 1994a, b; Stuefer 1996).

Since high degrees of plasticity are consistently associated with vertically oriented spacers (Huber 1997; this paper), it could be argued that differences in spacer plasticity between orthotropic and plagiotropic shoots are a side effect of differences in orientation. This would imply that spacer length responses are linked to the gravitropic response. However, indirect evidence that spacer plasticity is not primarily determined by direction of shoot growth comes from the hybrid Potentilla anglica (Huber 1995). This stoloniferous species, produced from a cross between a parent with an erect growth form and a parent with a stoloniferous growth form, has highly plastic internodes. Further experimental studies involving hybrids between erect and stoloniferous species may help to reveal the actual determinants of spacer plasticity in herbaceous plants.

\section{Plant growth}

Except for internode length, shading had far smaller effects on orthotropic than plagiotropic shoots. Parameters such as biomass and modular unit production, the mean weight of modular units and the size of leaves, did not respond significantly to shade in orthotropic shoots.

The fact that reducing PPFD by $76 \%$ did not affect biomass or modular unit production in orthotropic shoots suggests that there is a ceiling on investment in these shoots which is reached at a light intensity below the $100 \%$ treatment used in this study. However, internodes on orthotropic shoots showed marked elongation responses when shaded. This response can be expected to position leaves and flowers in the upper regions of the canopy. In intact clones the consequent enhanced light capture would probably be used to promote further vegetative growth (Suzuki and Hutchings 1997). In addition, shade-induced internode elongation in orthotropic shoots would elevate inflorescences in the canopy, and may thereby facilitate pollination and seed dispersal (van der Pijl 1969; Burrows 1973). This suggests that different selection pressures, namely enhanced light interception in horizontally oriented, vegetative shoots, and enhanced capacity to export photosynthate, increased 
pollination and greater seed dispersal in vertically oriented, generative shoots, may favour plasticity of vertical spacers in the two shoot types. These different factors lead to the convergent result of high degrees of shadeinduced elongation of vertically oriented spacers.

\section{Conclusions}

This study shows that within the same species homologous structures respond differently to shading if they are situated on orthotropic or plagiotropic shoots. Although the meristematic origin of orthotropic and plagiotropic shoots is identical, the ecological functions (vegetative or generative) and orientations (horizontal or vertical) of these shoots are different, resulting in considerable differences in numerous morphological and growth characters, and in their plasticity in response to light conditions.

Differences in the plasticity of internodes and petioles of vertically and horizontally oriented shoots imply that such traits are not species-specific, but that they can be altered during ontogeny. This may be due to benefits associated with plastic increases in the length of vertically oriented internodes in terms of light capture and pollination, but low or unpredictable net benefits from shade-induced elongation of horizontally oriented internodes. The regulation of this plasticity remains an intriguing topic for further research.

Acknowledgements We gratefully acknowledge the critical reading of a former version of this manuscript by Josef Stuefer, Marinus Werger and two anonymous referees. We thank Josef Stuefer for collecting the plant material in Hungary and for practical help during the experiment.

\section{References}

Ballaré CL (1994) Light gaps: sensing the light opportunities in highly dynamic canopy environments. In: Caldwell MM, Pearcy RW (eds) Exploitation of environmental heterogeneity by plants. Academic Press, London, pp 73-110

Bell AD (1984) Dynamic morphology: a contribution to plant population ecology. In: Dirzo R, Sarukhán J (eds) Perspectives on plant population ecology. Sinauer, Sunderland, pp 48-65

Burrows FM (1973) Calculating the primary trajectories of plumed seeds in steady wind with variable convection. New Phytol 72:647-664

Birch CPD, Hutchings MJ (1992) Analysis of ramet development in the stoloniferous herb Glechoma hederacea using a plastochron index. Oikos 63:387-394
Dong M (1993) Morphological plasticity of the clonal herb Lamiastrum galeobdolon (L.) Ehrend. \& Polatschek in response to partial shading. New Phytol 124:291-300

Dong M (1994) Foraging through morphological plasticity in clonal herbs. PhD dissertation, Utrecht University

Dong M (1995) Morphological responses to local light conditions in clonal herbs from contrasting habitats, and their modification due to physiological integration. Oecologia 101:282-288

Evans JP (1992) The effect of local resource availability and clonal integration on ramet functional morphology in Hydrocotyle bonariensis. Oecologia 89:265-276

Hegi G (1981) Illustrierte Flora von Mitteleuropa. Parey, Berlin

Huber H (1995) Growth form and plasticity of the hybrid Potentilla anglica and its two parent species. Abstr Bot 19:61-73

Huber H (1996) Plasticity of internodes and petioles in prostrate and erect Potentilla species. Funct Ecol 10:401-409

Huber H (1997) Architectural plasticity of stoloniferous and erect herbs in response to light climate. PhD Thesis, Utrecht University

Huber H, Fijan A, During, HJ (in press) A comparative study of spacer plasticity in erect and stoloniferous herbs. Oikos

Hutchings MJ, Kroon H de (1994) Foraging in plants: the role of morphological plasticity in resource acquisition. Adv Ecol Res 25:159-238

Kroon H de, Hutchings MJ (1995) Morphological plasticity in clonal plants: the foraging concept reconsidered. J Ecol 83:143152

Monsi M, Saeki T (1953) Über den Lichtfaktor in den Pflanzengesellschaften und seine Bedeutung für die Stoffproduktion. Jpn J Bot 14:22-52

Oborny B (1994a) Growth rules in clonal plants and environmental predictability - a simulation study. J Ecol 82:341-351

Oborny B (1994b) Spacer length in clonal plants and the efficiency of resource capture in heterogeneous environments: a Monte Carlo simulation. Fol Geobot Phytotax 29:139-158

Pijl L van der (1969) Principles of dispersal in higher plants. Springer, Heidelberg New York Berlin

Potvin C, Lechowicz MJ, Tardif S (1990) The statistical analysis of ecophysiological response curves obtained from experiments involving repeated measures. Ecology 71:1389-1400

SAS (1988) SAS/STAT users guide. SAS Institute, Cary

Scheiner SM (1993) Genetics and evolution of phenotypic plasticity. Annu Rev Ecol Syst 24:35-68

Sokal RR, Rohlf FJ (1995) Biometry: the principles and practice of statistics in biological research. Freeman, New York

Stuefer JF (1996) Potential and limitations of current concepts regarding the response of clonal plants to environmental heterogeneity. Vegetatio 127:55-70

Suzuki J, Hutchings MJ (1997) Interactions between shoots in clonal plants and the effects of stored resources. In: Kroon $\mathrm{H}$ de, Groenendael $\mathbf{J}$ van (eds) The ecology and evolution of clonal plants. Backhuys, Leiden, pp 311-329

Tappeiner U, Cernusca A (1996) Microclimate and fluxes of water vapour, sensible heat and carbon dioxide in structurally differing subalpine plant communities in the Central Caucasus. Plant, Cell and Environment 19:403-417

Thompson L (1993) The influence of natural canopy density on the growth of white clover, Trifolium repens. Oikos 67:321-324

Watkinson AR, White J (1986) Some life-history consequences of modular construction in plants. Philos Trans R Soc B 313:31-51 